TITLE:

\title{
Microwave Stark decelerator for polar molecules
}

$\operatorname{AUTHOR}(S)$ :

Enomoto, K; Momose, T

CITATION:

Enomoto, K ... [et al]. Microwave Stark decelerator for polar molecules. PHYSICAL REVIEW A 2005, 72(6): 061403.

ISSUE DATE:

2005-12

URL:

http://hdl.handle.net/2433/50039

RIGHT:

Copyright 2005 American Physical Society 
PHYSICAL REVIEW A 72, 061403(R) (2005)

\title{
Microwave Stark decelerator for polar molecules
}

\author{
Katsunari Enomoto and Takamasa Momose* \\ Department of Chemistry, Graduate School of Science, Kyoto University, Kyoto 606-8502, Japan
}

(Received 19 September 2005; published 30 December 2005)

\begin{abstract}
We propose a method to decelerate polar molecules from a beam using a microwave field. A moving standing wave of a microwave electric field causes an ac Stark shift to polar molecules and decelerates them. The method is applicable to polar molecules in rotational ground states and can be used to directly load a microwave trap. Numerical simulations are presented indicating large phase-space acceptance volume.
\end{abstract}

DOI: 10.1103/PhysRevA.72.061403

PACS number(s): 33.80.Ps, 33.55.Be, 39.10.+j

Currently, cooling and trapping of polar molecules have attracted keen interest from several research communities, ranging from particle to condensed matter physics [1]. For example, cold molecules generally allow high-resolution spectroscopy, aiding in the search for new physics; such is the case with searches for the permanent electric dipole moment of the electron [2] and parity violation in chiral molecules [3]. In the area of atomic physics, there is intense ongoing work to understand cold collisions and ultracold chemistry [4-6] as well as for the use of polar molecules as qubits in a quantum computer [7]. Finally, if the ultracold regime is achieved for polar molecules at high density, it is also predicted that different types of quantum degenerate matter may be produced due to strong, anisotropic, longrange dipole-dipole interactions [8,9].

Recently, several methods to generate cold or ultracold molecules have been experimentally demonstrated, although not all have been applied yet to polar molecules. One approach is to transform ultracold atoms into ultracold molecules using Feshbach resonance or photoassociation [6]. Another approach is to cool molecules directly from molecular beams using a cold buffer gas. Although this method is general and has been used very successfully to make large numbers of cold polar molecules, ultracold molecules have not yet been realized [10,11]. Alternatively, electrostatic Stark deceleration, which controls the velocity of a beam of polar molecules with a time-varying quasistatic electric field, has been used to decelerate several kinds of molecules $[12,13]$, bringing some into the ultracold regime. These decelerated molecules have been loaded into an electrostatic trap [12] and an ac electric trap [14]. Filtering and guiding slow molecules from an effusive source with an electrostatic field has also been performed [15]. An optical dipole force of an intense pulsed laser [16] and a collision with an inert gas beam [17] have also been utilized to create slow molecules. There are several other proposals to produce slow molecules using the optical dipole force [18-20]. Finally, the rotating jet is a versatile method to create a supersonic jet beam pulse that has the critical feature of being slow in the lab frame [21], but has so far not been used to load a trap because

\footnotetext{
*Present address: Department of Chemistry, The University of British Columbia, 2036 Main Mall, Vancouver, B.C., Canada V651Z1
}

velocities obtained so far have corresponded to energies much higher than currently available trap depths.

In the present paper, we propose a method to decelerate molecular beams from a rotating jet (or similar) source using microwave fields. The basic idea is to make a time-varying series of upward potential slopes by employing the ac Stark shift in a standing wave in a microwave cavity. The use of the ac Stark shift enables deceleration of a wide range of polar molecules because of easy tunability of microwave sources. Furthermore, some portion of the slowed molecules are trapped by the stationary microwave field after the deceleration is completed. Our proposed method builds on the studies of the dynamics of the electrostatic Stark decelerator developed by Bethlem et al. and Friedrich [22,23] and the work of DeMille et al. [24], the latter having suggested the microwave trap for polar molecules. Microwave traps can trap molecules in a high-field seeking state, which is the "true" ground state, so that inelastic two-body collisions can be completely suppressed [24]. Our proposed microwave slowing can be used with ground-state molecules. Here we work out in some detail both the idea and concrete realization of a microwave slower that should be suitable for loading microwave traps and other applications.

A proposed experimental setup is illustrated in Fig. 1. A pair of microwave mirrors create a Fabry-Perot resonant cavity. One of the mirrors has a small hole for introducing a molecular beam along the symmetry axis of the cavity. The molecular beam is assumed to be predecelerated by a rotating jet [21]. A high-power microwave generator with the capability of amplitude modulation in $\sim 10 \mathrm{kHz}$ drives the cavity. The time-varying potential that is created for the molecules is similar to that of the electrostatic Stark decelerator. For simplicity, we consider the case of a diatomic ${ }^{1} \Sigma$ state molecule with no hyperfine structure and a microwave field with linear polarization perpendicular to the cavity [25]. The

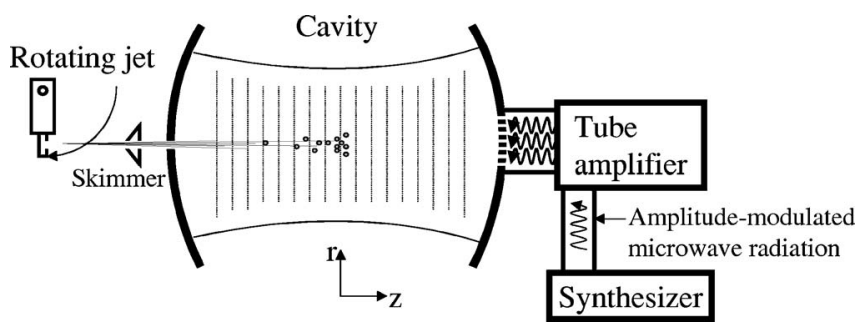

FIG. 1. Example of the experimental apparatus. 
decelerator is general, depending only on the existence of a dipole. Thus, as is the case with the electrostatic slower and microwave trap, it can be applied to many molecule types including $\Pi$ states, triplets, etc.

Our analysis of slowing begins by writing the rotational state as $|J, m\rangle$, where $J$ is the rotational quantum number and $m$ is the projection to the polarization axis $x^{\prime}$. If the frequency $\omega$ of the microwave field is close to the resonance frequency $\omega_{01}$ between the $J=1$ and $J=0$ states, a two-level approximation becomes a good assumption ignoring all rotational states other than the $J=1$ and 0 states. With the rotating wave approximation, the semiclassical Hamiltonian in the rotating frame with the frequency $\omega$ can be written as

$$
H=\Delta|1,0\rangle\langle 1,0|+\frac{\mu_{01} E}{2}(|1,0\rangle\langle 0,0|+| 0,0\rangle\langle 1,0|),
$$

where $\Delta=\hbar\left(\omega_{01}-\omega\right)$ is the detuning and $E$ is the amplitude of the microwave electric field. The symbol $\mu_{01}$ is the transition dipole moment defined as $\mu_{01}=\mu\left\langle 0,0\left|n_{x^{\prime}}\right| 1,0\right\rangle$, where $\mu$ is the dipole moment of the molecule and $n_{x^{\prime}}$ is the operator indicating the $x^{\prime}$ component of the unit vector along the molecular axis. From the relation $\left\langle J, m\left|n_{x^{\prime}}\right| J+1, m\right\rangle$ $=\sqrt{(J+m+1)(J-m+1) /(2 J+1)(2 J+3)}$, we obtain $\mu_{01}$ $=\mu / \sqrt{3}$. We only consider the red detuning case $(\Delta>0)$, and the ac Stark shift of the ground state is given by $\Delta / 2$ $-\sqrt{\Delta^{2}+\mu_{01}{ }^{2} E^{2}} / 2$. Note that molecules in a $\Sigma$ electronic state and in the rotational ground state often have a small dc Stark shift under a realistic experimental condition even if they have a large $\mu$. This is because the detuning $\Delta$ is equal to $\omega_{01}$ for a dc field and it is often larger than $\mu_{01} E$, since electric field strength has a practical limit not to cause a discharge. For example, in the electrostatic Stark deceleration experiment, the electric field strength used was typically $\sim 100 \mathrm{kV} / \mathrm{cm}$ [22]. Note also that the spontaneous decay rate of a rotational transition is so small that the eigenstate of Hamiltonian (1), in which the rotational excited state is mixed, has a long lifetime even if $\Delta$ is small.

We assume that the standing wave of the microwave field in the cavity is the lowest-order transverse mode $\left(\mathrm{TEM}_{00}\right)$. The electric field $E(z, r, t)$ is given by

$$
\begin{aligned}
E(z, r, t)= & \frac{E_{0}}{\sqrt{1+\left(\frac{z}{z_{0}}\right)^{2}}} \exp \left(-\frac{k r^{2}}{2 z_{0}\left[1+\left(\frac{z}{z_{0}}\right)^{2}\right]}\right) \cos (\omega t) \\
& \times \cos \left[-\tan ^{-1}\left(\frac{z}{z_{0}}\right)+\frac{k r^{2}}{2 z\left[1+\left(\frac{z_{0}}{z}\right)^{2}\right]}+k z\right],
\end{aligned}
$$

where $z$ and $r$ are displacements from the center of the cavity in the axial and the radial directions, respectively, $t$ is time, $z_{0}$ is the Rayleigh length, $k$ is the wave number, and $E_{0}$ is the amplitude at the center. We designate each set of an antinode and a node as a stage, and define $W_{i}$ as the potential depth of the $i$ th stage. If the microwave field is turned on and off when a molecule comes to antinodes and nodes, respectively, the molecule loses its kinetic energy by $\Sigma_{i} W_{i}$ during the flight.
To decelerate a finite portion of a molecular beam, however, the timing of the switching must be adjusted, so that leading molecules lose more kinetic energy than trailing molecules. For motion in the longitudinal direction along the cavity axis, "phase stability requirements" determine the $z-v_{z}$ phase-space area to which molecules to be decelerated belong [22,23]. In terms of the phase stability, there is a trade-off between this area and the total loss of kinetic energy. We define a phase $\phi$ as $90^{\circ}$ at nodes and $-90^{\circ}$ at antinodes, and designate a molecule whose phase is constant $\phi_{0}$ at the moment of switching off as a "synchronous" molecule. We approximate that the potential energy at $\phi$ is expressed by $W_{i}(\sin \phi-1) / 2$, which holds well especially in the case of $\Delta \gg \mu_{01} E$. The kinetic energy loss of the synchronous molecule at the $i$ th stage is then given by $W_{i} \sin \phi_{0}$. Under this approximation and the one that the time-dependent force is replaced with an average force, the longitudinal motion of a molecule at the $i$ th stage in the moving frame fixed to the synchronous molecule is described by an equation [22]

$$
\frac{d^{2} \delta \phi}{d t^{2}}=-\frac{8 \pi W_{i}}{M \lambda^{2}}\left[\sin \left(\phi_{0}+\delta \phi\right)-\sin \phi_{0}\right],
$$

where $M$ is the mass of the molecule, $\lambda$ is the wavelength of the microwave field, and $\delta \phi=4 \pi\left(z-z_{\text {sync }}\right) / \lambda$ with $z_{\text {sync }}$ the $z$ position of the synchronous molecule. This equation is analogous to the one for the motion of a pendulum with a constant torque, and there is an area in the phase space where $\delta \phi$ continues to oscillate without divergence. This area will be referred to as an acceptance area, and molecules within this area are decelerated simultaneously with the synchronous molecule. The boundary of the acceptance area is described by an equation [23]

$$
\begin{aligned}
\frac{d \delta \phi}{d t}= & \pm\left(\frac { 1 6 \pi W _ { i } } { M \lambda ^ { 2 } } \left[\cos \left(\phi_{0}+\delta \phi\right)+\cos \phi_{0}\right.\right. \\
& \left.\left.+\left(2 \phi_{0}+\delta \phi-\pi\right) \sin \phi_{0}\right]\right)^{1 / 2} .
\end{aligned}
$$

This equation indicates that the acceptance range of $\delta \phi$ is determined only by $\phi_{0}$, and the range of $d \delta \phi / d t$ is proportional to $\sqrt{W_{i} / M \lambda^{2}}$. Therefore, the acceptance range of $z$ per stage length is scaled by $\lambda$, and that of $v_{z}$ is scaled by $\sqrt{W_{i} / M}$. A comprehensive study on the dynamics of such a deceleration process is seen in Ref. [23].

The use of a cavity provides an enhanced standing wave of the microwave field. We assume a cavity with the quality factor $Q$ and the length $L$. The maximum value $E_{0, \max }$ of the electric field amplitude $E_{0}$ is given by $E_{0 \text {, max }}{ }^{2}=4 P Q / \pi \epsilon_{0} c z_{0} L$, where $P$ is the power of microwave radiation supplied by the microwave generator, $\epsilon_{0}$ is the permittivity of vacuum, and $c$ is the speed of light. Under the assumption that the wavelength $\lambda$ is much smaller than both $L$ and $z_{0}$, the total kinetic energy loss $W_{\text {tot }}$ during the flight between the mirrors of the cavity is estimated as

$$
W_{\text {tot }}=\frac{4 P Q \mu_{01}^{2} \tan ^{-1}\left(\frac{L}{2 z_{0}}\right) \sin \phi_{0}}{\pi \epsilon_{0} c L \lambda \Delta},
$$

in the limit $\Delta \gg \mu_{01} E$, and 


$$
W_{\text {tot }}=4 \mu_{01} \sinh ^{-1}\left(\frac{L}{2 z_{0}}\right) \sin \phi_{0} \sqrt{\frac{P Q z_{0}}{\pi \epsilon_{0} c L \lambda^{2}}},
$$

in the limit $\Delta \ll \mu_{01} E$. The following things should be noted. Although the reduction of $P$ can be compensated by decreasing $\lambda$ for constant $W_{\text {tot, }}$, it reduces the acceptance area that is proportional to $\lambda \sqrt{W_{i}}$. As for Eq. (6), maximizing $\sinh ^{-1}\left(L / 2 z_{0}\right) \sqrt{z_{0} / L}$ is not the best strategy, since small $z_{0}$ leads to small $\sqrt{W_{i}}$ near the mirrors and therefore limits the acceptance area.

In reality, the instantaneous switching of the microwave field assumed above must be replaced with smooth amplitude modulation. In terms of the quality factor $Q$, the modulation angular frequency $\Omega$ and $E_{0, \max }$ are in a trade-off relationship. The modulation frequency needed is $\Omega \simeq 2 \pi v / \lambda$ where $v$ is the initial velocity of the molecular beam, and it poses a limitation that $Q$ must be smaller than $\omega / \Omega$. Therefore the bandwidth of the microwave field should be as narrow as possible to use a high- $Q$ cavity. We describe the amplitude modulation with a function $f(t)$, so that the electric field amplitude is given by the product of $|f(t)|$ and $|E(z, r)|$ in Eq. (2) with dropping the high-frequency component $\cos \omega t$. An efficient and simple choice of $f(t)$ is that the phase of modulation is kept constant relative to that of the synchronous molecule, i.e.,

$$
f(t)=\cos \left[k z_{\mathrm{sync}}(t)-\tan ^{-1}\left(\frac{z_{\mathrm{sync}}(t)}{z_{0}}\right)-\frac{\phi_{0}^{\prime}}{2}\right],
$$

where $\phi_{0}^{\prime}$ is an arbitrary phase analogous to $\phi_{0}$ in Eqs. (3)-(6) though the switching is no longer instantaneous. In this case, the kinetic energy loss per stage is given by $2 W_{i} \sin \phi_{0}^{\prime} / 3$, and the estimation of $W_{\text {tot }}$ corresponding to Eq. (6) is given by

$$
W_{\text {tot }}=\frac{8}{3} \mu_{01} \sinh ^{-1}\left(\frac{L}{2 z_{0}}\right) \sin \phi_{0}^{\prime} \sqrt{\frac{P Q z_{0}}{\pi \epsilon_{0} c L \lambda^{2}}} .
$$

Many molecules are suitable for our method. Especially, a large- $\mu$ molecule containing a moderately light atom, such as $\mathrm{Li}, \mathrm{O}$, or $\mathrm{F}$, is favorable, because a large dipole moment simply enhances $W_{\text {tot }}$, and moderately high transition frequency (around several ten, of GHz) ensures the large number of deceleration stages and the availability of high-power microwave generators. Furthermore, small mass $M$ is preferable in the case where the molecules are involved in some carrier gas for the predeceleration.

In order to determine the acceptance volume of the decelerator, the transverse motion of the molecular beam needs to be considered. Qualitatively, if the wave plane of the standing wave is flat, molecules in the high-field seeking state experience force toward the center axis, as is understood from Eq. (2) since $\partial|E| / \partial r<0$ at $z=0$. This force makes a guiding field for the molecular beam. The curved wave plane near the mirrors degrades this guiding force. We were able to take these, and other, factors into consideration through a quantitative simulation. We have performed a threedimensional Monte Carlo simulation of the deceleration of a molecular beam using a specific test molecule, ${ }^{24} \mathrm{Mg}^{16} \mathrm{O}$. This was chosen as a "test" molecule because it has a large

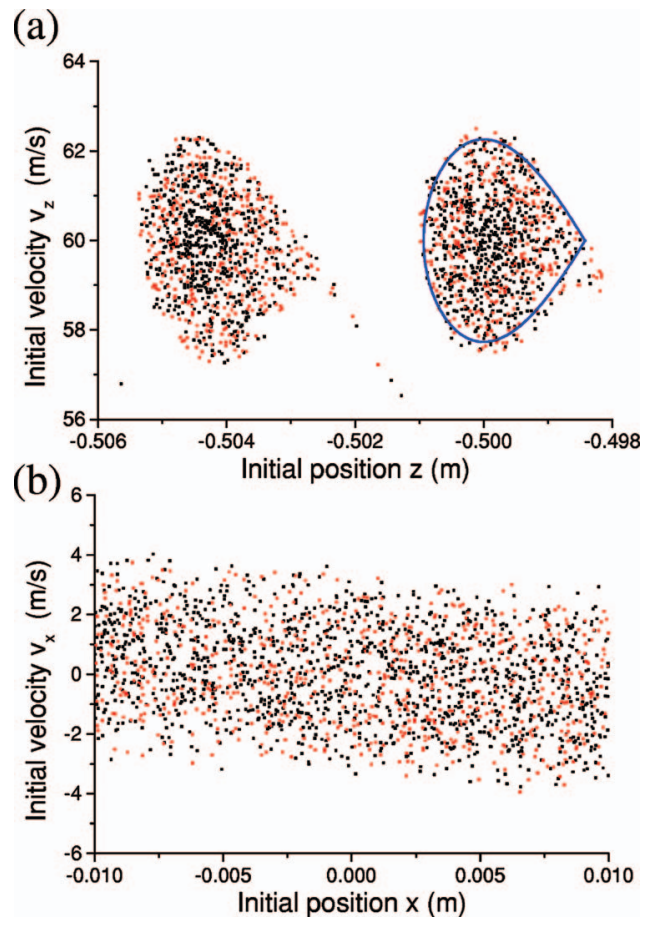

FIG. 2. (Color) Initial phase-space distribution of molecules to be decelerated and/or trapped, obtained from the Monte Carlo simulation. Red dots indicate molecules of the resultant energy $<0.2 \mathrm{~K}$, and black dots are the trapped molecules. (a) Projection onto the $z-v_{z}$ plane. The solid line is the expected boundary (see text). (b) Projection onto the $x-v_{x}$ plane.

dipole moment ( $\mu=6.2$ D) [26], no hyperfine structure, moderate transition frequency $\left(\omega_{01} / 2 \pi=34.290 \mathrm{GHz}\right)$ [27], and a simple $X^{1} \Sigma^{+}$ground state. The rotating jet method has successfully produced a beam of Xe carrier gas with the average velocity of $\sim 60 \mathrm{~m} / \mathrm{s}[21]$, and we assume that $\mathrm{MgO}$ molecules are mixed with this carrier gas. This situation corresponds to $\Omega / 2 \pi \simeq 6.9 \mathrm{kHz}$ and kinetic energy of $8.7 \mathrm{~K}$. The microwave source, such as a klystron or a traveling-wave tube, is assumed to have a power of $P=3.0 \mathrm{~kW}$. Other parameters are set to be $L=1.0 \mathrm{~m}, z_{0}=0.34 \mathrm{~m}, Q=10^{5}$, and $\Delta=1.0 \mathrm{GHz}$, all well within the range of available technology. The resultant potential depth is $0.26 \mathrm{~K}$ at the center of the cavity. The synchronous molecule is assumed to have an initial velocity of $\left(v_{x}, v_{y}, v_{z}\right)=(0,0,60) \mathrm{m} / \mathrm{s}$ and an initial position of $(x, y, z)=(0,0,-L / 2)$. The microwave field is modulated as indicated by Eq. (7). We ignored the gravity in this simulation, as estimates indicate it is a negligible effect.

The results of the simulation are as follows. In the case of $\phi_{0}^{\prime}=16^{\circ}$, the synchronous molecule is stopped at $z=L / 2$. The estimation of $W_{\text {tot }}$ obtained from Eq. (8) is $9.5 \mathrm{~K}$, which roughly agrees with the fact that the initial kinetic energy is $8.7 \mathrm{~K}$. Figure 2 shows a result with $\phi_{0}^{\prime}=25^{\circ}$. Deceleration is completed at $z=0.13 \mathrm{~m}$ and $t=18 \mathrm{~ms}$ in this case. Molecules in the phase space to be decelerated and/or trapped are plotted as projection onto the $z-v_{z}$ and $x-v_{x}$ planes. In Fig. 2, $5.4 \%$ of all molecules in the rectangular solid volume shown are decelerated so that the sum of the potential and kinetic energies becomes $<0.2 \mathrm{~K}$. Moreover, $3.5 \%$ of all molecules end up with "negative energy," which means they are trapped 
by the microwave field. In Fig. 2(a), there are two groups of decelerated molecules in this volume and they are separated by $\lambda / 2$. We have confirmed that by extending the initial range of $z$ in our simulations, about 20 adjacent groups contain comparable numbers of decelerated molecules. The solid line shows the expected acceptance area obtained from Eq. (4), in which the phase $\phi_{0}$ is replaced with $\phi_{0}^{\prime}$, and the potential depth $W_{i}$ was substituted by $2 / 3$ [due to the smooth modulation of Eq. (7)] of that at the position of the mirror since the smallest $W_{i}$ among all stages determines the resultant acceptance area. This theoretical boundary agrees well with the result of the numerical simulation. Figure 2(b) shows that only molecules with $\left|v_{x}\right| \lesssim 4 \mathrm{~m} / \mathrm{s}$ are decelerated. This is because the transverse guiding force is not so strong that the acceptance range of the transverse velocity is not large. In fact, oscillation in the transverse motion is only fewer than twice during deceleration. However, the acceptance range of the initial $x$ position is as large as $>20 \mathrm{~mm}$ because the beam cross section of the microwave field is larger than this $x$ displacement. Consequently, the six-dimensional acceptance volume is about $[2 \mathrm{~mm}($ per $\lambda / 2$ interval $) \times 4 \mathrm{~m} / \mathrm{s}] \times[>20 \mathrm{~mm}$ $\times 6 \mathrm{~m} / \mathrm{s}]^{2}$. This acceptance volume is enough for experi- mental implementation, compared with the electrostatic Stark decelerator [22].

In summary, we have discussed a method to decelerate polar molecules and to load them directly into a trap using a time-varying standing wave of a microwave field enhanced by a cavity. The dynamics of the longitudinal motion has been analyzed based on the theories developed for the electrostatic Stark decelerator $[22,23]$. We have determined the efficiency of the method with a simulation of deceleration of $\mathrm{MgO}$ molecules from a pulsed beam source. The sixdimensional acceptance volume is comparable to that of an electrostatic Stark decelerator. Approximately half of molecules in the volume are trapped after deceleration. We expect that the same idea will be applicable to the frequency ranges other than microwave. For example, an intense infrared $\mathrm{cw}$ laser switched by an acousto-optic modulator may work similarly when the frequency is tuned close to a vibrational resonance.

We are grateful to T. Yabuzaki, M. Kumakura, and J. Doyle for helpful discussions. We acknowledge support from the Japan Society for the Promotion of Science.
[1] J. Doyle, B. Friedrich, R. V. Krems, and F. Masnou-Seeuws, Eur. Phys. J. D 31, 149 (2004).

[2] J. J. Hudson, B. E. Sauer, M. R. Tarbutt, and E. A. Hinds, Phys. Rev. Lett. 89, 023003 (2002).

[3] M. Ziskind, C. Daussy, T. Marrel, and Ch. Chardonnet, Eur. Phys. J. D 20, 219 (2002).

[4] N. Balakrishnan and A. Dalgarno, Chem. Phys. Lett. 341, 652 (2001).

[5] R. V. Krems and A. Dalgarno, J. Chem. Phys. 120, 2296 (2004).

[6] K. Burnett, P. S. Julienne, P. D. Lett, E. Tiesinga, and C. J. Williams, Nature (London) 416, 225 (2002).

[7] D. DeMille, Phys. Rev. Lett. 88, 067901 (2002).

[8] K. Goral, L. Santos, and M. Lewenstein, Phys. Rev. Lett. 88, 170406 (2002).

[9] M. A. Baranov, M. S. Mar'enko, Val. S. Rychkov, and G. V. Shlyapnikov, Phys. Rev. A 66, 013606 (2002).

[10] J. D. Weinstein et al., Nature (London) 395, 148 (1998).

[11] S. E. Maxwell et al., Phys. Rev. Lett. 95, 173201 (2005).

[12] H. L. Bethlem et al., Nature (London) 406, 491 (2000).

[13] H. L. Bethlem, A. J. A. van Roij, R. T. Jongma, and G. Meijer, Phys. Rev. Lett. 88, 133003 (2002).

[14] J. van Veldhoven, H. L. Bethlem, and G. Meijer, Phys. Rev. Lett. 94, 083001 (2005).

[15] S. A. Rangwala, T. Junglen, T. Rieger, P. W. H. Pinkse, and G.
Rempe, Phys. Rev. A 67, 043406 (2003).

[16] R. Fulton, A. I. Bishop, and P. F. Barker, Phys. Rev. Lett. 93, 243004 (2004).

[17] M. S. Elioff, J. J. Valentini, and D. W. Chandler, Science 302, 1940 (2003).

[18] P. F. Barker and M. N. Shneider, Phys. Rev. A 66, 065402 (2002).

[19] G. Dong, W. Lu, and P. F. Barker, Phys. Rev. A 69, 013409 (2004).

[20] B. Friedrich, Phys. Rev. A 61, 025403 (2000).

[21] M. Gupta and D. Herschbach, J. Phys. Chem. A 105, 1626 (2001).

[22] H. L. Bethlem, F. M. H. Crompvoets, R. T. Jongma, S. Y. T. van de Meerakker, and G. Meijer, Phys. Rev. A 65, 053416 (2002).

[23] B. Friedrich, Eur. Phys. J. D 31, 313 (2004).

[24] D. DeMille, D. R. Glenn, and J. Petricka, Eur. Phys. J. D 31, 375 (2004).

[25] If a multiphoton transition causes a problem, circular polarization must be used as pointed out in Ref. [24].

[26] H. Büsener, F. Heinrich, and A. Hese, Chem. Phys. 112, 139 (1987).

[27] T. C. Steimle, Y. Azuma, and P. G. Carrick, Astrophys. J. Lett. 277, L21 (1984). 